\title{
Properties of Confined Polymer Melts
}

\author{
A. SIKORSKI* \\ Department of Chemistry, University of Warsaw \\ Pasteura 1, 02-093 Warsaw, Poland
}

(Received November 19, 2004)

\begin{abstract}
Properties of simple models of confined linear polymer chains were studied by means of the Monte Carlo method. Model chains were built of united atoms (statistical segments) and embedded to a simple cubic lattice. Then polymers were put into a slit formed by two parallel impenetrable surfaces. Chain lengths were varied up to 800 segments and the density of the polymer melt was changed up to 0.5. A Metropolis-like sampling Monte Carlo algorithm was used to determine the static properties of this model. The influence of the size of the confinement, the polymer melt concentration and the chain length on the chain's size and the structure was studied. The universal behavior of all confined polymer linear chains under consideration was found and discussed.
\end{abstract}

PACS numbers: 02.50.Ng, 36.20.Ey, 61.25.Hq

\section{Introduction}

The properties of polymers in confined space are the subject of many studies because of their practical importance in lubrication, adhesion, separation methods based on the capillary electrophoresis corrosion protecting coating or colloidal stabilization [1]. These systems are difficult to be studied experimentally although recently a progress has been made. The questions concerning the influence of the confinement on the properties of polymers or proteins are especially interesting from the theoretical point of view [2].

Recently, some computer simulation studies were published on confined polymer systems [3-7]. It was shown that the dynamic properties of confined polymer melts are significantly different from those of bulk polymers. The scaling behavior static and dynamic of chain's properties, internal structure of chains, and instan-

*e-mail: sikorski@chem.uw.edu.pl 
taneous shape were intensively studied and discussed. This was caused by the fact that confined polymer chains were deformed and thus were in a thermodynamically unfavorable state so they exerted strong pressure on the surfaces. What is interesting, these differences exist even for relatively short chains which are not entangled. The determination of all the details concerning the structure of layers formed by confined chains is important for studying the dynamic properties.

The influence of the internal macromolecular architecture (linear chains, star-branched chains, cyclic chains) on the properties of confined chains was also studied employing simple lattice models and computer simulation [8-10]. The shortand long-time dynamics of confined chains were determined [8, 9]. The orientation and the deformation of coils were also studied. Instantaneous shape of the confined chains was also determined [10].

In this paper we present the results of computer simulations of simplified models of confined linear chain systems. Thus, the main goal of this study was to study the static properties of the linear chain systems. We compared properties of relatively dense polymer melts and infinitely diluted solution linear chains both located between a pair of two impenetrable walls.

\section{The model}

A polymer chain was in our model reduced to a united atom sequence. Each model chain consisted of the sequence of $N$ identical united atoms (beads) connected by $N-1$ segments (homopolymer) [10]. Beside single chains (infinite dilution) we also simulated dense polymer melts consisting of $n$ chains each of equal length $N$. We studied the properties of a chain as a whole without details on the level of a bead so this model was quite sufficient for this purpose [11]. In order to make the calculation more efficient the chains were embedded to a simple cubic lattice. The excluded volume interactions were introduced into the model by forbidding the chain to cross itself and the chains were fully flexible with no local stiffness introduced. No long-range attractive potential was added which corresponded to good solvent conditions (or a high temperature). The chains were put into a slit formed by a pair of two impenetrable parallel surfaces and the distance between these surfaces $d$ was varied (there were $d$ unoccupied lattice sites between walls in the direction perpendicular to the walls). The confining walls were repulsive only and thus their influence was entropic only. Periodic boundary conditions were imposed in directions along the surfaces only. The size of the Monte Carlo box $L$ was chosen to be large enough to avoid the interaction of a chain with itself. The concentration of the melt was expressed as the fraction of the accessible volume in the slit occupied by polymer beads

$$
\varphi=\frac{N m}{L^{2} d}
$$

where $m$ stands for the number of chains. 


\section{The simulation method}

The properties of model systems were determined by means of the Monte Carlo simulation. The sampling algorithm consisted of local random modifications of chain's conformation [8]. These modifications were: 2-bond motion, 3-bond motion, 3-bond crankshaft motion, 1-bond end of chain reorientations, and 2-bond end of chain reorientations $[8,12]$. A new conformation was accepted due to geometrical constraints and the excluded volume. Initial configurations of the system were built in the process of simultaneous growing and equilibration of chains in order to obtain the desired chain lengths and polymer densities [8, 11]. Simulations were started from these prepared systems for given distances between the surfaces and the production run for each distance $d$ was done separately. For each system under consideration $(N, n, \varphi)$ we performed 20-30 independent simulation runs using different initial configurations.

\section{Results and discussion}

The simulations were performed for the chains built of $N=50,100,200$, 400 , and 800 beads. The choice of chain length was done in order to cover the regions where chains were self-entangled and entangled [11]. For the melt studied the number of chains was not constant in order to maintain the constant density of the melt. The distance between the surfaces $d$ was varied between 30 and 3 in order to cover the regime from almost not distorted chain to a flattened 2-dimensional chain. (One has to remember that the distance $d=3$ is the smallest at which changes of chain's conformations are possible to occur in our model [8].) The size of the Monte Carlo box along the two remaining directions was changed in such a way as to maintain the constant concentration of the polymer segments in the cell. For this purpose, the edge of the Monte Carlo box was changed between 43 and 136 and the number of chains was varied between 550 and 562 (for chains $N=50$ ), 275 and 281 (for chains $N=100$ ), 137 and 140 (for chains $N=200$ ), 68 and 70 (for chains $N=400$ ), 34 and 35 (for chains $N=800$ ). Additionally, the simulations were carried out for melt densities $\varphi$ between 0.1 and 0.5 . The calculations for higher densities cannot be carried out as the sampling algorithm appeared to be inefficient for these systems.

The first question concerned the changes of the mean size of linear macromolecules in the confinement. The size of a chain is usually described by the mean-squared radius of gyration $\left\langle S^{2}\right\rangle$, i.e. the mean-squared distance of a bead from the center-of-mass. In Fig. 1 we present double logarithmic plot of $\left\langle S^{2}\right\rangle$ as a function of the length of chain $N$. One can observe that for all confined polymer systems under consideration the size of chains scales as $N^{\gamma}$. For single chains the scaling exponent changes from $1.17 \pm 0.01$ (for almost an unconfined chain) to $1.48 \pm 0.01$ (for a strongly confined chain). For the dense melt this exponent was almost constant: $\gamma=0.99 \pm 0.02$ and $1.01 \pm 0.01$ for $d=30$ and 3, respectively. 
These results are not unexpected because for unconfined chains: $\gamma=6 / 5$ for single polymers in 3 dimensions, $\gamma=3 / 2$ for single polymers in 2 dimensions and $\gamma=1$ for melts. We would like to emphasize that the above scalings indicate that strongly confined linear polymers have properties of two-dimensional chains.

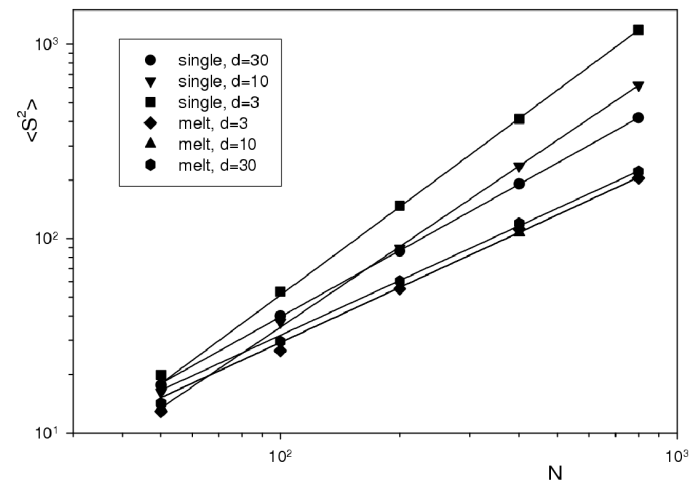

Fig. 1. The mean-square radius of gyration $\left\langle S^{2}\right\rangle$ as a function of the chain length $N$ for some distances between the walls $d$ for single chain and for the melt with the segment density $\varphi=0.5$. The distances between the surfaces $d$ are given in the inset.

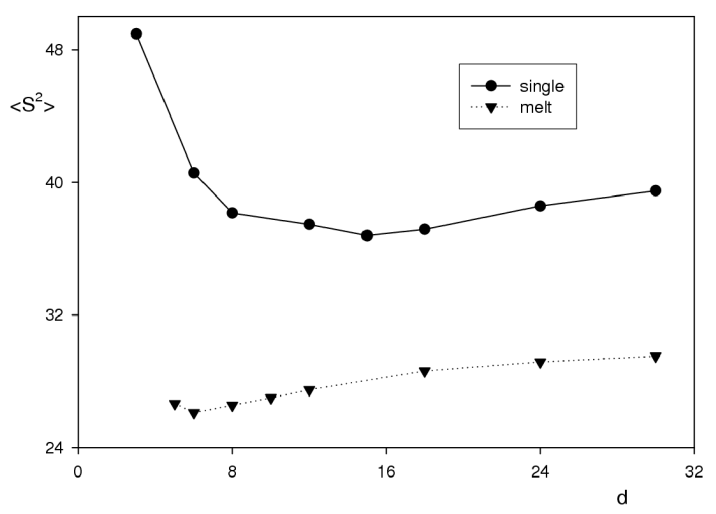

Fig. 2. The mean-square radius of gyration $\left\langle S^{2}\right\rangle$ as a function of the distance between the walls $d$ for the chain length $N=100$.

Figure 2 presents changes of $\left\langle S^{2}\right\rangle$ with the distance between the confining surfaces $d$ for a single chain with $N=100$ beads and for a melt consisting of such chains at the density $\varphi=0.5$. One can observe that the changes of the mean-squared radius of gyration are similar in both cases: the size of the chain decreases slightly when the distance $d$ decreases. Then, the size starts to grow rapidly. The shape of $\left\langle S^{2}\right\rangle$ curve can be explained by the fact that during the squeezing of chains the $z$-contribution to the radius of gyration decreases while the $x y$-contribution 
decreases. A minimum on the $\left\langle S^{2}\right\rangle$ curve corresponds to the distance between the walls which is close to a size of unconfined polymer chains $[8,10]$.

It was previously shown that the size of confined star-branched and linear chains can be described by a universal curve $[3,8]$. Hence, we tried to check if this universal behavior can also be extended to polymer melts. The size is now described by a reduced quantity $\left\langle S^{2}\right\rangle /\left\langle S_{0}^{2}\right\rangle$ where the index " 0 " denotes an unconfined (free) chain. For a single chain $\left\langle S_{0}^{2}\right\rangle$ corresponds to the mean-squared radius of gyration of a macromolecule consisting of the same number of segments but unconfined, i.e. located in the Monte Carlo box with periodic boundary conditions imposed in all directions. For the melt case we assumed that $\left\langle S_{0}^{2}\right\rangle$ corresponded to the mean-squared radius of gyration of chains in unconfined melt of the same density. The reduced size of the slit $d^{*}$ was defined as

$$
d^{*}=d /\left\langle S_{0}^{2}\right\rangle^{1 / 2} \text {. }
$$

Figure 3 presents the plots of the radius of gyration, the same as in Fig. 1 but using the reduced quantities. Other chain lengths were also added for the test of the concept. One can observe that for a single chain regardless its length these reduced values are located approximately on the same curve. Data concerning melts are also located on the same master curve. The deviations from that curve are larger in the case of the longest chains and for the confinements $d^{*}<1$. This means that for longer chains the impact of the confinement on polymer size was stronger. This can be explained by the fact that the conformational changes and the reorientation of longer chains and dense system is more difficult although it is difficult to judge if it is a result of the inefficiency of the sampling algorithm.

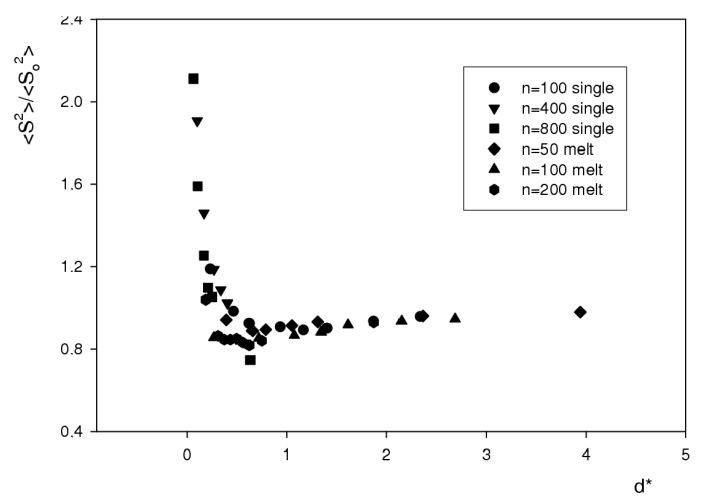

Fig. 3. The reduced mean-square radius of gyration $\left\langle S^{2}\right\rangle /\left\langle S_{0}^{2}\right\rangle$ as a function of the reduced distance between the walls $d^{*}$. The chain lengths are given in the inset.

The ratio $\left\langle S^{2}\right\rangle /\left\langle R^{2}\right\rangle$ is the next commonly used parameter that describes the size and the structure of polymer chains. The dependence of this ratio on the size of the slit $d$ is shown in Fig. 4. The ratios do not change monotonically: for 


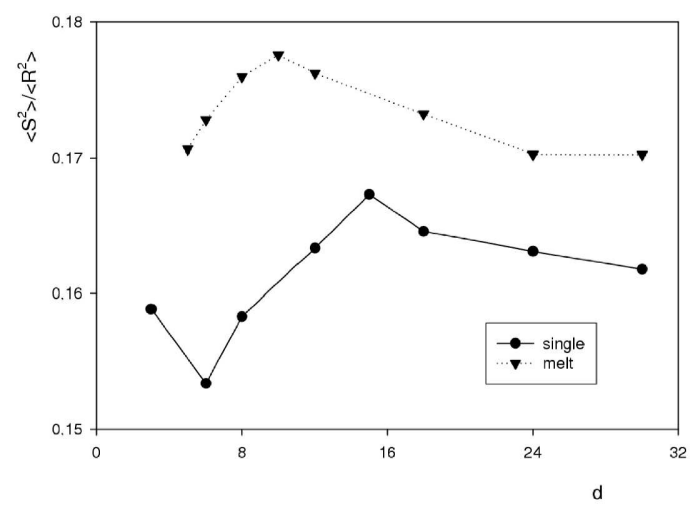

Fig. 4. The ratio $\left\langle S^{2}\right\rangle /\left\langle R^{2}\right\rangle$ as the function of the distance between the walls $d$ for the chain length $N=100$.

a single chain one can observe a maximum for the same size of the slit for which a minimum on the $\left\langle S^{2}\right\rangle$ curve was found (see Fig. 2). For the melt case there is a maximum on the curve but is located for larger $d$ when compared to the $\left\langle S^{2}\right\rangle$ curve. The ratio for the melt is always considerably larger when compared to a single chain. The value of this ratio found for a single linear chain without the excluded volume was 0.167 while the introduction of the excluded volume led to the ratio $0.157[1,11]$.

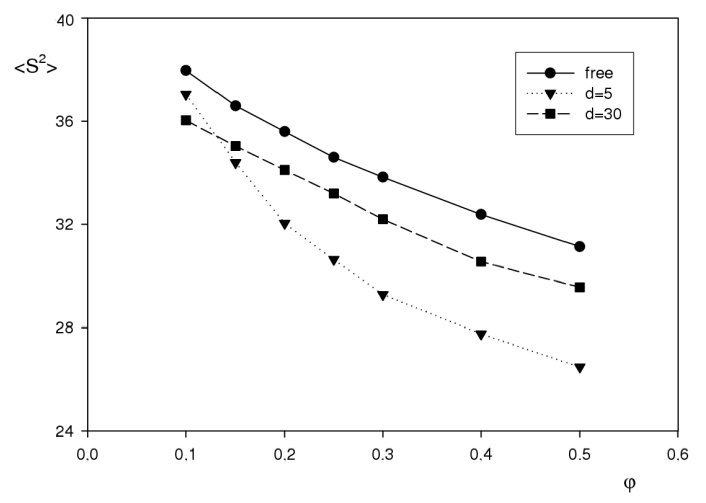

Fig. 5. The mean-square radius of gyration $\left\langle S^{2}\right\rangle$ as a function of the polymer density $\varphi$ in the slit. The case of the melt consisting of linear chains with $N=100$ beads for the distances between the surfaces $d=5$ and $d=30$ and for a free melt.

The changes of chains size with the polymer density $\varphi$ are presented in Fig. 5. One can observe that in general the size of chains decreases with the increase in the density and the shape of both curves is similar. This behavior is qualitatively the same as for free (unconfined) melts, while for more squeezed chains $(d=5)$ the decrease is more rapid [4]. For densities below 0.3 the decrease in the 


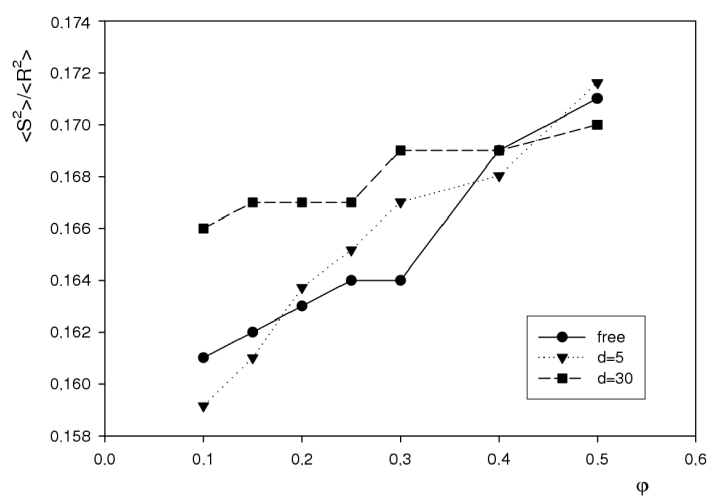

Fig. 6. The ratio $\left\langle S^{2}\right\rangle /\left\langle R^{2}\right\rangle$ as the function of the polymer density $\varphi$ in the slit. The case of the melt consisting of linear chains with $N=100$ beads for the distances between the surfaces $d=5$ and $d=30$.

chains' size is more rapid for smaller size of the slit while for larger densities the changes are similar. Figure 6 presents the changes of the ratio $\left\langle S^{2}\right\rangle /\left\langle R^{2}\right\rangle$ with the polymer density $\varphi$. Here one can notice that the behavior of this parameter is quite different in both cases under consideration. In general, the ratio increases with the density and the increase is more rapid for the more confined chains. For the larger slit, the ratio is rather close to the theoretical value 0.167 for chains without excluded volume. For a squeezed melt the ratio is close to 0.157 - a value that is characteristic of unconfined chains with the excluded volume.

The structure of confined polymer systems can also be described by the distribution of polymer beads along the direction perpendicular to the confining surfaces. Figure 7 presents a typical example of the density profiles for single chains and melts. The shape of the density profiles for single chains is Gaussian.

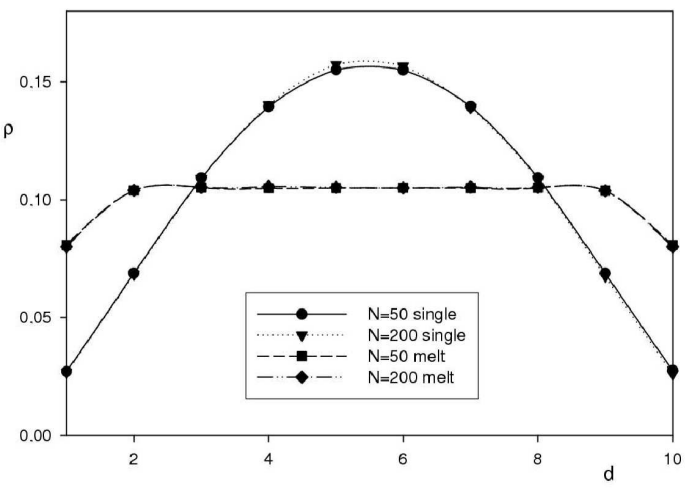

Fig. 7. Segment densities along the $z$-axis for the single chains and melt. The case of the distance $d=10$. The chain lengths are given in the inset. 
For melts, one can see the constant polymer density across the slit. The only exception was the segment densities for the layers closest to the confining surfaces which were considerably smaller. This is obviously the effect of a repulsive entropic force caused by the excluded volume effect. The density profile for all chain lengths are exactly the same for melts. The density profiles for other distances $d$ are very similar to those discussed above.

\section{Conclusions}

Properties of confined linear polymer chains were studied using simple and reduced lattice models. The behavior of single linear chains and of melts composed of such chains was determined. Polymer chains were constructed as a sequence of identical segments on a simple cubic lattice at good solvent conditions (no energetical contact was distinguished). The Metropolis-like Monte Carlo simulation algorithm used enables us to study long chains up to 800 statistical segments and melts with the density of polymer segments 0.5 .

It was shown that regardless of the melt density and the chain length the changes of polymer's size can be described by a universal curve. The scaling of the polymer size depends on the degree of the confinement: strongly confined chains behave as two-dimensional ones.

One has to remember that interaction of polymers with a surface (like adsorption) is one of the most important factors that influence the dynamic properties of confined polymers. Therefore, the further extension of this model should include polymer bead-surface interactions which is underway.

\section{References}

[1] K. Eisenriegler, Polymers Near Surfaces, World Scientific, Singapore 1993.

[2] H.-W. Sun, S. Granick, Science 258, 1339 (1992).

[3] G. ten Brinke, D. Ausserre, G. Hadziioannou, J. Chem. Phys. 89, 4374 (1988).

[4] A. Milchev, K. Binder, Eur. Phys. J. B 3, 477 (1998).

[5] Y. Yoshida, Y. Hiwatari, Molec. Simul. 22, 91 (1999).

[6] D.V. Kuznetsov, A.C. Balazs, J. Chem. Phys. 113, 2479 (2000).

[7] N. Fatkulin, R. Kimmich, E. Fischer, C. Mattea, U. Beginn, M. Kroutieva, New J. Phys. 6, art. no 46 (2004).

[8] J. Skolnick, A. Kolinski, Adv. Chem. Phys. 78, 223 (1990).

[9] A. Sikorski, P. Romiszowski, J. Chem. Phys. 116, 1731 (2002).

[10] A. Sikorski, P. Romiszowski, J. Chem. Phys. 120, 7206 (2004).

[11] A. Sikorski, Acta Phys. Pol. A 103, 339 (2003).

[12] P.H. Verdier, W.H. Stockmayer, J. Chem. Phys. 36, 227 (1962). 